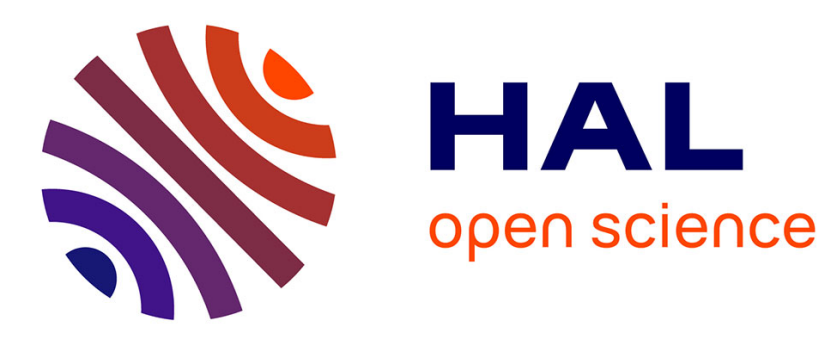

\title{
Integral method to study transition radiation from surfaces with arbitrary profile
}

Y. Takakura, Olivier Haeberlé

\section{To cite this version:}

Y. Takakura, Olivier Haeberlé. Integral method to study transition radiation from surfaces with arbitrary profile. Physical Review E: Statistical, Nonlinear, and Soft Matter Physics, 2000, 61 (4), pp.4441-4443. 10.1103/PhysRevE.61.4441 . hal-00958575

\section{HAL Id: hal-00958575 \\ https://hal.science/hal-00958575}

Submitted on 12 Mar 2014

HAL is a multi-disciplinary open access archive for the deposit and dissemination of scientific research documents, whether they are published or not. The documents may come from teaching and research institutions in France or abroad, or from public or private research centers.
L'archive ouverte pluridisciplinaire HAL, est destinée au dépôt et à la diffusion de documents scientifiques de niveau recherche, publiés ou non, émanant des établissements d'enseignement et de recherche français ou étrangers, des laboratoires publics ou privés. 


\title{
Integral method to study transition radiation from surfaces with arbitrary profile
}

\author{
Y. Takakura \\ Laboratoire des Systèmes Photoniques, ENSPS, Université Louis Pasteur, Boulevard Sébastien Brant, F-67400 Illkirch, France \\ O. Haeberlé \\ Groupe LABEL, Equipe EEA, Université de Haute-Alsace, IUT, 61 rue Albert Camus, F-68093 Mulhouse Cédex, France
}

(Received 21 September 1999)

\begin{abstract}
Transition radiation is produced when an electron moving at constant speed crosses the boundary separating two media with different optical properties. We propose an alternative description of this phenomenon, based on an integral representation of the electromagnetic field and show that the approach gives known results for a flat surface. Compared to previously published methods, based either on the reciprocity theorem in electromagnetism or on a four-dimensional Fourier representation of the field generated by the moving electron, this method has the advantage that it can be applied to general boundary geometries.
\end{abstract}

PACS number(s): 41.60.-m, 42.25.Gy, 42.25.Fx

Transition radiation is produced when a charged particle crosses the interface separating media with different optical properties [1]. Two different approaches have been proposed so far for flat surfaces. One consists in writing the incident field as a superposition of virtual photons which are scattered at the interface, giving birth to propagative waves which constitute the transition radiation spectrum $[2,3]$. The total field must then satisfy the boundary conditions at the interface. While of general validity, this method gives easy-tosolve boundary problems for flat surfaces only. Another approach consists in making use of the reciprocity theorem in electromagnetism: one solves a reciprocal problem in which real waves interact with the surface and the electron $[4,5]$. Because real waves only are taken into account, for a flat surface the introduction of the Fresnel coefficients is straightforward. Very few works have been dedicated to the study of transition radiation from rough surfaces [6]. For a periodic profile, van den Berg [7] made use of an integral method to describe the radiation emitted when an electron passes close (but without hitting it) and parallel to a surface grating, known as the Smith-Purcell radiation. In this paper, we give an approach to the transition radiation, based on an integral representation of the electromagnetic field similar to the one proposed by van den Berg. Figure 1 describes the configuration we consider, with an electron crossing the interface separating vacuum from a perfectly conducting medium and the so-called in plane radiation only. The origin $O$ of the $(x, y, z)$ reference frame attached to the surface corresponds to the impact point. The direction of incidence is determined by the angle $\Phi$ between the electron trajectory and the $x$ axis. The photon escape direction is defined by the angle $\theta$ with respect to the $z$ axis. The $(X, Y=y, Z)$ reference frame attached to the electron trajectory will be used for mathematical proofs (Fig. 2).

As in Ref. [7], the electron is modeled as a charged material point with a charge $q$ and travels with constant velocity $v_{0}$. Such a motion generates an electric current density $J$ given by

$$
J(X, Y=y, Z, t)=q v_{0} \delta\left(X-v_{0} t, Z\right) u_{X},
$$

which in turn is responsible for the total electromagnetic field $(E, H)$ described by the following Maxwell equations (in S.I. units):

$$
\begin{gathered}
\nabla \times E=-\frac{\partial B}{\partial t}, \\
\nabla \times H=J+\frac{\partial D}{\partial t},
\end{gathered}
$$

with $B=\mu_{0} H$ and $D=\epsilon_{0} E$. One introduces the Fourier integral [7]:

$$
\begin{aligned}
U(x, y, z, t)= & \left(2 \pi^{2}\right)^{-1} \operatorname{Re}\left[\int_{0}^{+\infty} d \omega \int_{-\infty}^{+\infty} \mathcal{U}(x, z ; \beta, \omega)\right. \\
& \times \exp (i \beta y-i \omega t) d \beta]
\end{aligned}
$$

for $E(x, y, z, t), H(x, y, z, t), J(x, y, z, t)$ and shows that the $(x, z)$ components of the Fourier variables $\mathcal{E}, \mathcal{H}$ of the electromagnetic field can be expressed in terms of its sole $y$ component which satisfies the Helmholtz equations:

$$
\partial_{x}^{2} \mathcal{E}_{y}+\partial_{z}^{2} \mathcal{E}_{y}+\left(k_{0}^{2}-\beta^{2}\right) \mathcal{E}_{y}=\left(\mu_{0} / \epsilon_{0}\right)^{1 / 2}\left(\beta / k_{0}\right) \partial_{x} \mathcal{J}_{x},
$$

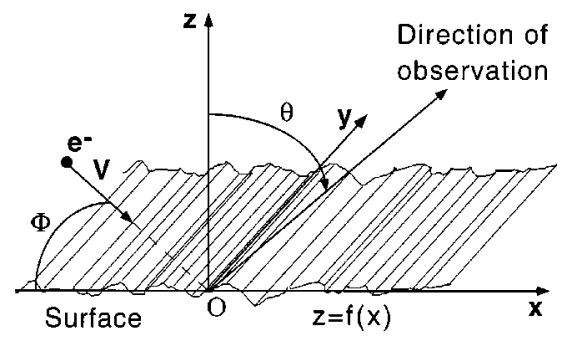

FIG. 1. Scheme of the emission of transition radiation as an electron hits a perfectly conducting surface. The trajectory of the particle lies in the $(x, z)$ plane and the incidence direction is determined by angle $\Phi$. The direction of observation is defined by angle $\theta$. The origin of the reference frame is at the impact point. 


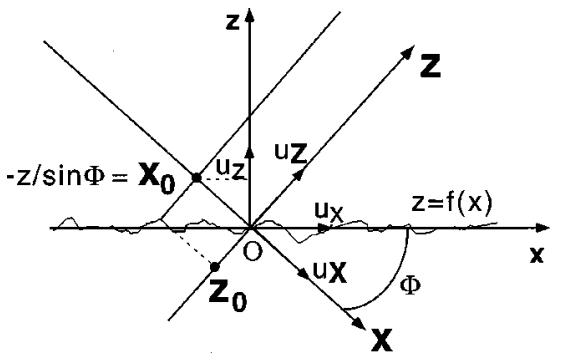

FIG. 2. Coordinate transformation $\left(u_{x}, u_{z}\right) \rightarrow\left(u_{X}, u_{Z}\right)$ used to derive an integral representation of the excitation field $u^{(i)}$. In order to take into account the sign of $z+X^{\prime} \sin \Phi$, the integral along $X^{\prime}$ is split into an integral from $-\infty$ to $-z / \sin \Phi$ and an integral from $-z / \sin \Phi$ to 0 .

$$
\partial_{x}^{2} \mathcal{H}_{y}+\partial_{z}^{2} \mathcal{H}_{y}+\left(k_{0}^{2}-\beta^{2}\right) \mathcal{H}_{y}=-\partial_{z} \mathcal{J}_{x}
$$

with $k_{0}=\omega / c, c$ being the velocity of light in vacuum.

In Ref. [7], the above equations are used to calculate the so-called incident field $\left(\mathcal{E}^{(i)}, \mathcal{H}^{(i)}\right)$ generated by the particle in motion and the solution of the radiation problem is treated as a grating problem [8] with an evanescent wave $\left(\mathcal{E}^{(i)}, \mathcal{H}^{(i)}\right)$ as the excitation field, the particle being neglected. In the present case, since the electron hits the surface, an interaction between the charge in motion and the interface should take place. As a result, the expression of the field $\left(\mathcal{E}^{(i)}, \mathcal{H}^{(i)}\right)$ as given in Ref. [7] and which has been calculated for a charge in the free space, is no longer valid. However, we shall show now that the actual transition radiation problem can be reduced to a boundary value problem similar to the Smith-Purcell problem [7] but with a different excitation field.

In the plane $(x, y=0, z)$ of incidence, since the wave vector has no component on the $y$ axis so that $\beta$ is equal to zero [7], the set of Eqs. (5), (6) reduces to

$$
\begin{gathered}
\partial_{x}^{2} \mathcal{E}_{y}+\partial_{z}^{2} \mathcal{E}_{y}+k_{0}^{2} \mathcal{E}_{y}=0, \\
\partial_{x}^{2} \mathcal{H}_{y}+\partial_{z}^{2} \mathcal{H}_{y}+k_{0}^{2} \mathcal{H}_{y}=-\partial_{z} \mathcal{J}_{x} .
\end{gathered}
$$

Here, the $y$ component of the electric excitation field $\mathcal{E}_{y}^{(i)}(x, z ; 0, \omega)$ vanishes and the magnetic excitation field $\mathcal{H}^{(i)}(x, z ; 0, \omega)$ has a sole component on the $y$ axis: the waves are TM polarized [7]. Hence, in such a plane, if we set $u(x, z)=\mathcal{H}_{y}(x, z ; 0, \omega)$, we have to find a field $u$ that satisfies the (inhomogeneous) Helmholtz equation:

$$
\Delta u+k_{0}^{2} u=-\partial_{z} \mathcal{J}_{x},
$$

a radiation condition as $z \rightarrow+\infty$ and the Neumann boundary condition:

$$
\frac{d u}{d n}(x, f(x))=0
$$

Here $z=f(x)$ represents the surface profile. Such a problem is well known in electromagnetism [9] and becomes a boundary value problem if one introduces the Green function $G$ :

$$
\Delta G+k_{0}^{2} G=-4 \pi \delta\left(x-x^{\prime}, z-z^{\prime}\right),
$$

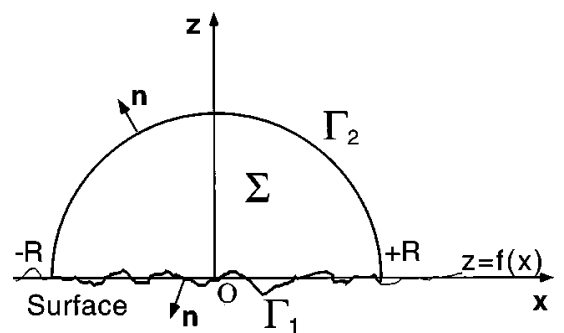

FIG. 3. Contour of integration for the Green theorem. As $R \rightarrow$ $+\infty$, the integral on $\Gamma_{2}$ vanishes because of the outgoing condition on $G$.

which is nothing else than the field created by an elementary point source located at $\left(x^{\prime}, z^{\prime}\right)$. Multiplying Eq. (9) by $G\left(x-x^{\prime}, z-z^{\prime}\right)$, Eq. (11) by $u\left(x^{\prime}, z^{\prime}\right)$, and substracting the resulting equations lead to

$$
\begin{aligned}
& \iint_{\Sigma} d x^{\prime} d z^{\prime}[G \Delta u-u \Delta G]+\iint_{\Sigma} d x^{\prime} d z^{\prime} G \partial_{z} \mathcal{J}_{x} \\
& =4 \pi u(x, z) \text { if } z>f(x) \\
& =0 \text { if } z<f(x)
\end{aligned}
$$

where $\Sigma$ is a closed surface bounded by the contour $\Gamma=\Gamma_{1}$ $+\Gamma_{2}$ (Fig. 3). Applying the second Green identity [10] to Eqs. (12), (13) and taking into account that $G$ must satisfy a radiation condition, one gets

$$
\begin{aligned}
u^{(i)}+\frac{1}{4 \pi} \int_{\Gamma_{1}} d l^{\prime}\left[G \frac{\partial u}{\partial n}-u \frac{\partial G}{\partial n}\right] & =u(x, z) \text { if } z>f(x) \\
& =0 \text { if } z<f(x)
\end{aligned}
$$

with

$$
\begin{gathered}
\frac{\partial}{\partial n}=n \nabla^{\prime}, \\
u^{(i)}(x, z)=\frac{1}{4 \pi} \iint_{\Sigma} d x^{\prime} d z^{\prime} G\left(x-x^{\prime}, z-z^{\prime}\right) \partial_{z} \mathcal{J}_{x} .
\end{gathered}
$$

From a physical point of view, Eqs. (14), (15) express the fact that, under the stress $u^{(i)}$, the charges on the boundary $\Gamma_{1}$ act in such a way as to extinguish the field in $z<f(x)$ : this is usually referred to as the extinction theorem [11]. From Eq. (17), we shall seek an integral representation of $u^{(i)}$. Noticing from Fig. 2 that $\left(u_{x}, u_{z}\right)$ can be deduced from $\left(u_{X}, u_{Z}\right)$ by a $\Phi$ rotation, one has

$$
\partial_{z} \mathcal{J}_{x}(X, Z)=q \exp \left(i \alpha_{0} X\right) \delta^{\prime}(Z)
$$

with $\alpha_{0}=k_{0} c / v_{0}$. As a consequence, Eq. (17) becomes

$$
\begin{aligned}
u^{(i)}(x, z)= & \frac{q}{4 \pi} \iint_{\Sigma} d X^{\prime} d Z^{\prime} \exp \left(i \alpha_{0} X^{\prime}\right) \delta^{\prime}\left(Z^{\prime}\right) \\
& \times G\left(x-X^{\prime} \cos \Phi-Z^{\prime} \sin \Phi, z\right. \\
& \left.+X^{\prime} \sin \Phi-Z^{\prime} \cos \Phi\right)
\end{aligned}
$$




$$
\begin{aligned}
= & \frac{q}{4 \pi} \int_{-\infty}^{0} d X^{\prime} \exp \left(i \alpha_{0} X^{\prime}\right) \int_{Z_{0}}^{+\infty} d Z^{\prime} \delta^{\prime}\left(Z^{\prime}\right) \\
& \times G\left(x-X^{\prime} \cos \Phi-Z^{\prime} \sin \Phi, z+X^{\prime} \sin \Phi-Z^{\prime} \cos \Phi\right),
\end{aligned}
$$

$Z_{0}$ being given in Fig. 2. Since according to the theory of distributions $[12]\left\langle\delta^{\prime}, \varphi\right\rangle=-\left\langle\delta, \varphi^{\prime}\right\rangle$, one gets

$$
\begin{aligned}
u^{(i)}(x, z)= & -\left.\frac{q}{4 \pi} \int_{-\infty}^{0} d X^{\prime} \exp \left(i \alpha_{0} X^{\prime}\right) \frac{d}{d Z^{\prime}}\right|_{Z^{\prime}=0} \\
& \times G\left(x-X^{\prime} \cos \Phi-Z^{\prime} \sin \Phi, z\right. \\
& \left.+X^{\prime} \sin \Phi-Z^{\prime} \cos \Phi\right) .
\end{aligned}
$$

Now, one considers the plane wave (Weyl) expansion [13] of $G$ :

$$
G(x, z)=i \int_{-\infty}^{+\infty} \frac{d \alpha}{\gamma} \exp (i \alpha x+i \gamma|z|)
$$

with $\alpha^{2}+\gamma^{2}=k_{0}^{2}$, so that

$$
\begin{aligned}
u^{(i)}(x, z)= & -\frac{q}{4 \pi} \int_{-\infty}^{+\infty} d \alpha \exp (i \alpha x) \\
& \times \int_{-\infty}^{0} d X^{\prime}\left[\frac{\alpha}{\gamma} \sin \Phi+\operatorname{sign}\left(z+X^{\prime} \sin \Phi\right) \cos \Phi\right] \\
& \times \exp \left[i\left(\alpha_{0}-\alpha \cos \Phi\right) X^{\prime}+i \gamma\left|z+X^{\prime} \sin \Phi\right|\right]
\end{aligned}
$$

In order to take the sign of $z+X^{\prime} \sin \Phi$ into account, one splits the integral along $X^{\prime}$ and gets finally

$$
\begin{aligned}
u^{(i)}(x, z)= & -\frac{q}{4 \pi}\left\{\exp \left(-i \alpha_{0} / \sin \Phi z\right) \int_{-\infty}^{+\infty} \frac{d \alpha}{\gamma}\right. \\
& \times \exp [i \alpha(x+z \cot \Phi)] \\
& \times\left[\frac{\alpha \sin \Phi-\gamma \cos \Phi}{i\left(\alpha_{0}-\alpha \cos \Phi-\gamma \sin \Phi\right)}\right. \\
& \left.-\frac{\alpha \sin \Phi+\gamma \cos \Phi}{i\left(\alpha_{0}-\alpha \cos \Phi+\gamma \sin \Phi\right)}\right]+\int_{-\infty}^{+\infty} \frac{d \alpha}{\gamma} \\
& \times \exp (i \alpha x+i \gamma z) \\
& \left.\times\left[\frac{\alpha \sin \Phi+\gamma \cos \Phi}{i\left(\alpha_{0}-\alpha \cos \Phi+\gamma \sin \Phi\right)}\right]\right\} \text { for } z>0 .
\end{aligned}
$$

To obtain Eq. (24) one uses the fact that $v_{0} / c$ is strictly smaller than 1 and the Fourier transform of the Heaviside step function

$$
\int_{0}^{+\infty} d t \exp (-i \omega t)=\mathrm{P}\left(\frac{1}{i \omega}\right)+\pi \delta(\omega)
$$

with P denoting the Cauchy principal value.
Finally, Eqs. (10), (14), and (15) lead to the following integral equation:

$$
u^{(i)}(x, z)-\frac{1}{4 \pi} \int_{\Gamma_{1}} d l^{\prime} u\left(x^{\prime}, f\left(x^{\prime}\right)\right) \frac{\partial G}{\partial n}=u(x, z) \text { if } z>f(x)
$$

$$
=0 \text { if } z<f(x) \text {, }
$$

where $u^{(i)}$ is given by Eq. (24). This integral equation is to be solved to obtain the total field $u(x, z)$, from which the radiative part constitutes the transition radiation spectrum.

We shall make a few comments about Eq. (24). First, the excitation field $u^{(i)}$ has been written as a superposition of a couple of integrals. The first integral presents a singularity on $z=-x \tan \Phi$ which indeed is the trajectory of the electron. Since its integrand does not satisfy the Helmholtz equation, such an integral represents a nonradiative component $u^{(i)}$. In the second integral, one recognizes a plane wave expansion: it corresponds to the progressive waves generated by a charge in motion which is suddenly stopped when it arrives onto the surface [14]. Second, a usual Neumann boundary condition is considered but in the present case, the particle actually hits the surface and creates a singularity on the impact point $O$. However, the extinction theorem tells us that Eq. (15) is also valid for $z<f(x)$. If one introduces a singular component in the integral and sets it equal to the opposite of the singularity of $u^{(i)}$, both of the singularities would cancel each other on the profile $z=f(x)$ and a regular boundary condition can actually be considered. When the surface is flat and perfectly conducting, a classical approach consists in involving the image charge: the singular component is then the image of the particle in motion. For a nonflat surface, such a classical approach cannot be used anymore and the singular component in that case should be called the generalized image of the particle in motion.

In order to prove the validity of the above investigations, we now calculate the power emitted when an electron hits a perfect plane $[f(x)=0]$. The limit equation for Eq. (26) as $z$ is closed to $f(x)$ is [9]:

$$
\frac{1}{2} u(x, f(x))=u^{(i)}(x, f(x))-\frac{1}{4 \pi} \int_{\Gamma_{1}} d l^{\prime} u\left(x^{\prime}, f\left(x^{\prime}\right)\right) \frac{\partial G}{\partial n} .
$$

On the surface, Eq. (28) reduces to $u(x, 0)=2 u^{(i)}(x, 0)$ so that the solution of the radiation problem for a perfect plane is

$$
u(x, z)=u^{(i)}(x, z)-\frac{1}{2 \pi} \int_{-\infty}^{+\infty} d x^{\prime} u^{(i)}\left(x^{\prime}, 0\right) \frac{\partial G}{\partial n}\left(x-x^{\prime}, z\right) .
$$

Recalling Eq. (22), one gets 


$$
\frac{\partial G}{\partial n}\left(x-x^{\prime}, z\right)=-\int_{-\infty}^{+\infty} d \alpha \exp \left[i \alpha\left(x-x^{\prime}\right)+i \gamma z\right] \text { for } z>0
$$

and finally

$$
\begin{aligned}
u(x, z)= & u^{(n r)}(x, z)-\frac{q}{4 \pi} \int_{-\infty}^{+\infty} \frac{d \alpha}{\gamma} \exp (i \alpha x+i \gamma z) \\
& \times\left[\frac{\alpha \sin \Phi-\gamma \cos \Phi}{i\left(\alpha_{0}-\alpha \cos \Phi-\gamma \sin \Phi\right)}\right. \\
& \left.+\frac{\alpha \sin \Phi+\gamma \cos \Phi}{i\left(\alpha_{0}-\alpha \cos \Phi+\gamma \sin \Phi\right)}\right] \text { for } z>0,
\end{aligned}
$$

where $u^{(n r)}$ corresponds to the nonradiative component of $u^{(i)}$ given by the first integral in Eq. (24). It is worth noting from Eq. (31) that the radiative component of $u(x, z)$ written as a plane wave expansion is the result of an interference between the radiative component [the second integral of Eq. (24)] of $u^{(i)}$ and the field emitted by the surface under the stress of the incoming component of the same field $u^{(i)}$. From an opticist's point of view, this is a very unusual scattering situation. However, this result clearly recalls the classical description of transition radiation in which the radiative field is written as a superposition of a direct field and a field reflected by the perfectly conducting surface $[4,5,14]$.

Now, the saddle point method [15] is used to express the integral in Eq. (31) in the far field so that the scattered power is proportional to

$$
\begin{aligned}
& \left|u^{(s c)}(r, \theta)\right|^{2} \\
& \quad=\left|\frac{q}{\sqrt{2 \pi k_{0} r}} \frac{\beta_{0} \sin \Phi\left(\sin \theta-\beta_{0} \cos \Phi\right)}{\left(1-\beta_{0} \cos \Phi \sin \theta\right)^{2}-\beta_{0}^{2} \cos ^{2} \theta \sin ^{2} \Phi}\right|^{2}
\end{aligned}
$$

with $\beta_{0}=v_{0} / c$. The angular factor in Eq. (32) is the same as the expression obtained in Refs $[2-5,14]$ for a perfectly conducting surface and confirms the validity of the approach presented in this paper.

Transition radiation has been investigated from a theoretical point of view. From Maxwell equations, we have reduce the problem to a boundary value problem. The resulting integral equation can be solved by means of known (numerical) tools. Peculiar aspects of such a radiation problem are the excitation field generated by the motion of the particle and, since the particle hits the surface, a singularity on the profile $z=f(x)$. This difficulty can be overcome, as the surface is perfectly conducting, by stating that the particle has a generalized image through the interface. Both particles cancel as they meet at the impact point $O$ and a regular boundary condition can be used. Applying the formalism in the case of a plane interface gives known expression of the scattered power, confirming at least in such a simple case, the validity of the investigations presented here.

Finally, it must be emphasized that Eq. (28) is valid whatever the profile $z=f(x)$, provided that the second derivative $f^{\prime \prime}(x)$ exists [8]. Our formalism can therefore be applied when a surface with an arbitrary profile is considered, for example a grating [16].

The authors gratefully acknowledge J. Harthong, P. Rullhusen, N. Maene, and P. Henri for valuable discussions which led to this work.
[1] I. F. Frank and V. L. Ginzburg, J. Phys. (Moscow) 9, 353 (1945)

[2] V. E. Pafomov, Proc. Lebedev Inst. of Physics 44, 25 (1971).

[3] M. L. Ter-Mikaelian, High Energy Electromagnetic Processes in Condensed Media (John Wiley \& Sons, New York, 1972).

[4] V. L. Ginzburg and V. N. Tsytovitch, Transition Radiation and Transition Scattering (Adam Higen, Bristol, 1990).

[5] P. Rullhusen et al., Novel Radiation Sources Using Relativistic Electrons (World Scientific, Singapore, 1998).

[6] R. A. Bagiyan and T. M. Ter-Mikaelyan, Zh. Eksp. Teor. Fiz, 81, 1249 (1981) [Sov. Phys. JETP 54, 666 (1981)].

[7] P. M. van den Berg, J. Opt. Soc. Am. 63, 1588 (1973).

[8] R. Petit, Electromagnetic Theory of Gratings (SpringerVerlag, Berlin, 1980).
[9] J. van Bladel, Electromagnetic Fields (McGraw-Hill, New York, 1964).

[10] P. M. Morse and H. Feshbach, Methods of Theoretical Physics (McGraw-Hill, New York, 1953).

[11] J. J. Sein, Opt. Commun. 2, 170 (1970).

[12] L. Schwartz, Mathematics for Physical Sciences (AddisonWesley, London, 1967).

[13] M. Nieto-Vesperinas and N. Garcia, Opt. Acta 28, 1651 (1981).

[14] L. Wartski, Thèse de Doctorat d'Etat, Université Paris-Sud, 1976.

[15] J. A. Kong, Electromagnetic Wave Theory (John Wiley \& Sons, New York, 1986).

[16] P. Henri et al., Phys. Rev. E 60, 6214 (1999). 